\title{
Specimen retrieval method using a sliding overtube for large colorectal neoplasm following endoscopic submucosal dissection
}
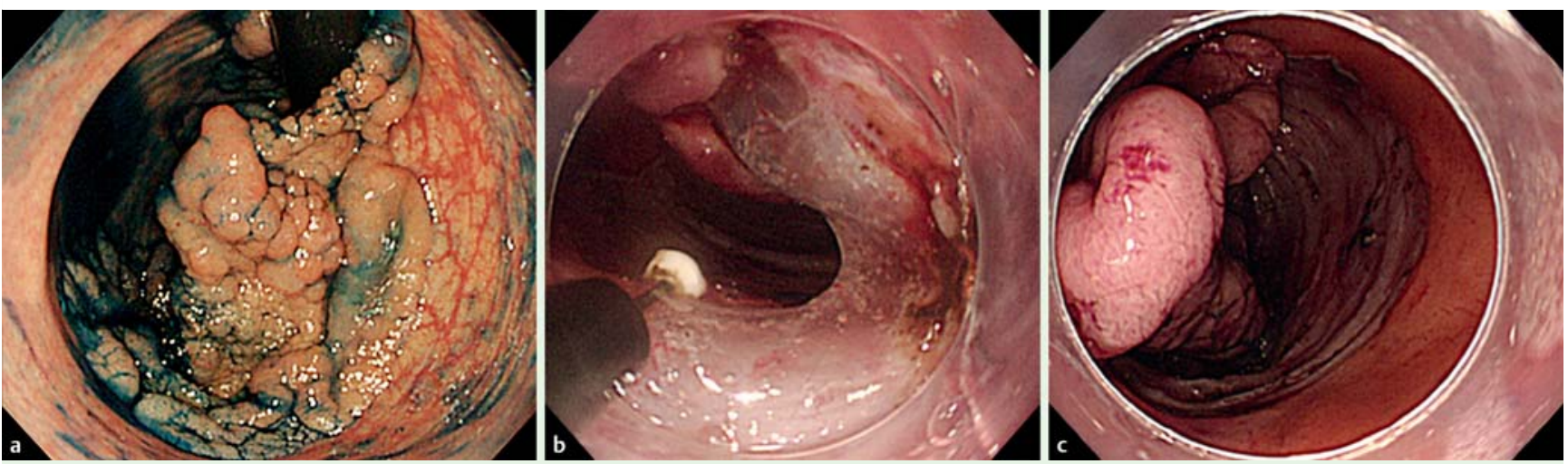

Fig. 1 Endoscopic submucosal dissection (ESD) of a large laterally spreading tumor (granular-type) of the rectum. a Colonoscopy revealed the tumor. b, $\mathbf{c}$ The ESD procedure was performed using a B-knife (Zeon Medical Co., Tokyo, Japan) and an IT knife (Olympus Co., Tokyo, Japan).
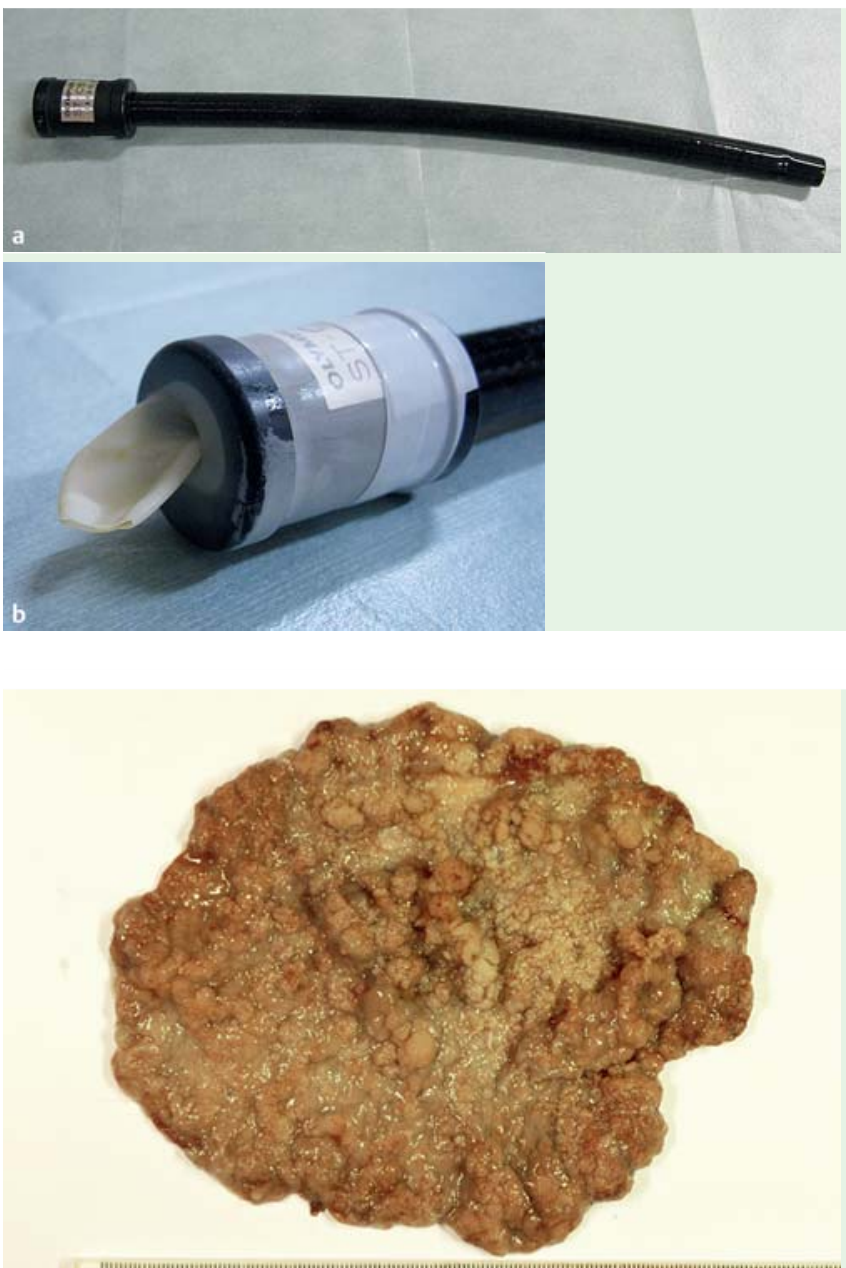

1
Fig. 2 A sliding tube technique was used to retrieve the large resection specimen. a The sliding tube (ST-C5; Olympus Co., Tokyo, Japan).

b The sliding tube was packed using the finger part of a surgical glove in order to increase the suction when placed over the colonoscope.

Fig. 3 The resected specimen was $135 \mathrm{~mm}$ long and $105 \mathrm{~mm}$ wide.
A 76-year-old woman was referred to the National Cancer Center Hospital for treatment of a large laterally spreading tumor (granular-type) of the rectum ( $\bullet$ Fig. 1 a). The lesion was $>10 \mathrm{~cm}$ in diameter. There were no endoscopic findings suggesting submucosal invasion, and therefore endoscopic submucosal dissection (ESD) was chosen as radical treatment. The ESD procedure was performed, and a en bloc resection was achieved ( $\bullet$ Fig. $\mathbf{1}$ b, c).

Following the ESD procedure, attempts were made to remove the specimen through the anal canal using a retrieval device (Roth Net; Olympus Co., Tokyo, Japan). However, this approach failed because of the size of the tumor. It was therefore decided to use a sliding overtube (ST-C5; Olympus Co., Tokyo, Japan) to remove the specimen from the rectum ( $\bullet$ Fig.2). To obtain maximal suction effect, one end of the overtube was packed using a surgical glove ( $\bullet$ Fig. 2 b). The colonoscope was withdrawn, and the overtube was fitted to the colonoscope. After visualizing the specimen, the overtube was carefully inserted into the rectum. Then the colonoscope was pulled out and back into the sliding tube repeatedly. Under direct visualization the specimen was vacuumed up into the sliding tube, and the sliding tube was removed from the rectum ( Video 1 ). The resected specimen was $135 \mathrm{~mm}$ long and $105 \mathrm{~mm}$ wide ( Fig.3). 
Histopathological examination revealed intramucosal carcinoma with tumor-free resection margins.

ESD allows the removal of large endoluminal gastrointestinal tumors, resulting in high rates of cure $[1,2]$. An en bloc resection specimen is needed for accurate pathological evaluation. In gastric and esophageal ESD, large resected specimens can be retrieved through the esophageal tract using large forceps because the tissue is thick and tough. Colorectal tumors, however, are fragile. Furthermore, the anal sphincter creates a collapsing pres-

\section{Video 1}

Endoscopic submucosal dissection (ESD) of a large laterally spreading tumor (granular-type) of the rectum. The large resection specimen was retrieved using an overtube, after standard methods using a Roth Net (Olympus Co., Tokyo, Japan) failed. sure, often impeding the removal of lesions from inside the rectum. The sliding tube and surgical glove are very common items in the endoscopy unit. Thus, the retrieval method described here can be performed very easily without additional specialized equipment.

Endoscopy_UCTN_Code_TTT_1AO_2AN

Competing interests: None

\section{Hisatomo Ikehara',2, Yutaka Saito², Toshio Uraoka ${ }^{3}$, Takahisa Matsuda², Hiroto Miwa ${ }^{1}$}

${ }^{1}$ Division of Gastroenterology, Hyogo

College of Medicine, Hyogo, Japan

2 Division of Endoscopy, National Cancer Center Hospital, Tokyo, Japan

${ }^{3}$ Department of Gastroenterology, National Hospital Organization Tokyo Medical Center, Tokyo, Japan

\section{References}

1 Saito Y, Uraoka T, Matsuda T et al. Endoscopic treatment of large superficial colorectal tumors: a case series of 200 endoscopic submucosal dissections. Gastrointest Endosc 2007; 66: 966-973

2 Fuishiro M, Yahagi N, Kakushima N et al. Outcomes of endoscopic submucosal dissection for colorectal epithelial neoplasms in 200 consecutive cases. Clin Gastroenterol Hepatol 2007; 5: 678-683

Bibliography

Dol http://dx.doi.org/

10.1055/s-0034-1391496

Endoscopy 2015; 47: E168-E169

(C) Georg Thieme Verlag KG

Stuttgart · New York

ISSN 0013-726X

Corresponding author

Yutaka Saito, MD, PhD

Endoscopy Division

National Cancer Center Hospital

5-1-1 Tsukiji, Chuo-ku

Tokyo 104-0045

Japan

Phone: +81-3-3542-2511

ytsaito@ncc.go.jp 